\title{
Drugs, Environment, Brain, and Behavior
}

\author{
Hans S. Crombag and Terry E. Robinson \\ University of Michigan, Ann Arbor
}

\begin{abstract}
The effects of psychoactive drugs are not just a function of their pharmacological actions, but are due to complex interactions among pharmacological, psychological, and environmental factors. We discuss here how drug-environment interactions determine the likelihood that addictive drugs produce a persistent form of neurobehavioral plasticity (sensitization) thought to be involved in the pathophysiology of addiction and relapse.
\end{abstract}

KEYWORDS_sensitization; neuroplasticity; addiction; relapse; genes

In humans, the behavioral and subjective effects of drugs vary enormously, even within the same individual, because drug effects are due to complex interactions among pharmacological, psychological, and environmental factors-for instance, whether a drug is taken in a drug-associated environment (e.g., a "crack house"), whether drug paraphernalia (e.g., syringes, pipes) are present, and what expectation the individual has about the drug. That many Vietnam combat troops who became dependent on heroin while in Vietnam discontinued use upon their return to the United States illustrates the influence of environmental factors on drug use.

Early laboratory studies on drug-environment interactions typically used operant conditioning procedures to study the effects of different schedules of reinforcement (i.e., variations in when and how often behavior is reinforced). For example, in pigeons, the anesthetic pentobarbital either increases or decreases bar pressing to obtain food, depending on the schedule of reinforcement in place at the time the drug is administered. An especially compelling demonstration of how even a simple manipulation of environmental context can influence drug effects is the observation that the lethal dose of amphetamine in mice varies up to 10 -fold depending on whether the drug is given in a large or small test cage, or to singly- or group-housed animals (see Kelleher \& Morse, 1968, for reviews of the early literature).

Nonetheless, there has been very little work on how drug-environment interactions influence the behavioral and neurobiological effects of drugs of abuse, and there has been even less consideration of drug-environment interactions when drugs are administered repeatedly, as would be the case in developing addicts. In this article, we focus on how environmental conditions in which drugs are ex-

Address correspondence to Hans S. Crombag or Terry E. Robinson, Department of Psychology, The University of Michigan, Ann Arbor, MI 48109-1109; e-mail: hcrombag@psy.jhu.edu or ter@umich.edu. perienced modulate long-lasting changes in the brain (i.e., neuroplasticity) that are caused by drug exposure-changes that are thought to be important in the transition to addiction and in relapse.

\section{DRUGS CHANGE THE BRAIN}

When a drug is taken repeatedly, some of its effects may decrease, or show tolerance, while other effects increase, or show sensitization. Historically, tolerance, and its role in the development of physical dependence, has been a central focus of research on addiction. However, increasing evidence suggests that long-lasting neurobiological changes related to sensitization play a critical role in addiction (Robinson \& Berridge, 2003). This is in part because the behavioral and psychological drug effects that undergo sensitization include effects on motor activation (psychomotor sensitization), incentive motivation ${ }^{1}$ (incentive sensitization), and the ability of drugs to sustain or reinforce drug-taking behavior (sensitization of drug reward). All of these behaviors and psychological processes are mediated in part by a neural circuit that involves the neurotransmitter dopamine and connects to many forebrain structures, in particular, the dorsal striatum, nucleus accumbens, prefrontal cortex, and related neural circuitry. Indeed, it is generally recognized that these dopamine circuits are crucial for sensorimotor function and for the reward value of natural stimuli, such as food, drink, and sex, as well as drugs. It is not surprising, therefore, that sensitization-related neuroplastic changes have been found in many components of this circuitry, and it is hypothesized that these brain changes may render individuals hypersensitive to the incentive motivational effects of drugs and thereby contribute to the transition to addiction (Robinson \& Berridge, 2003). Therefore, identifying factors that influence susceptibility to sensitization may help researchers understand some of the variability in susceptibility to drug addiction and relapse.

\section{ENVIRONMENTAL MODULATION OF BEHAVIORAL SENSITIZATION}

In a series of experiments, we and our colleagues have studied the influence of environmental context on the ability of drugs to produce psychomotor sensitization (Badiani, Browman, \& Robinson, 1995). In these experiments, rats receive repeated injections of a drug in one of two conditions: either where they live (home condition) or in a distinct

${ }^{1}$ Incentive motivation refers to the psychological process by which objects, events, places, and their mental representations become attractive and wanted (incentive salience) and thereby acquire the ability to control goal-directed behavior. 


\section{Environmental modulation of sensitization}
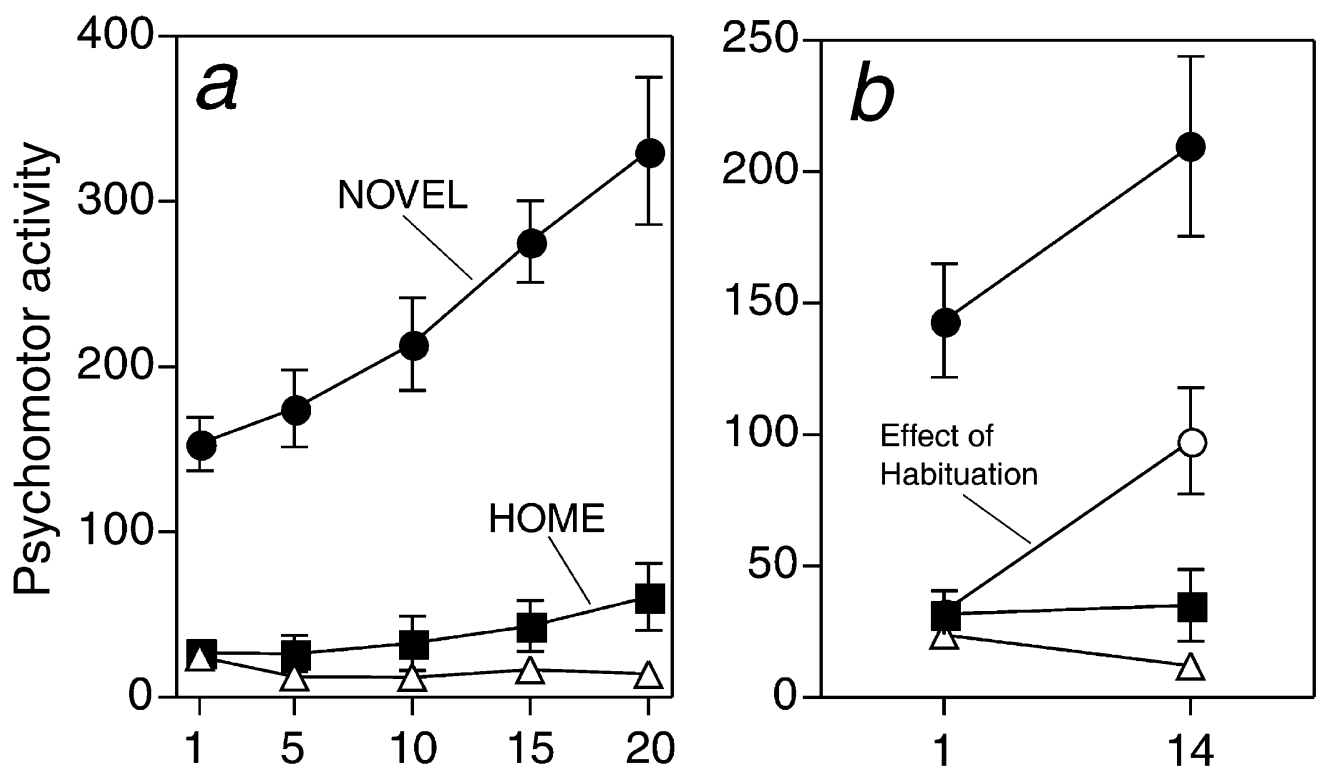

\section{Injection number}

Fig. 1. Results of two independent studies in rats investigating the ability of environmental context to modulate the development of psychomotor sensitization. The level of psychomotor activity is graphed as a function of the number of unsignaled injections of amphetamine. The graph in (a) shows the psychomotor response by rats that received 20 injections ( 1 injection each day) of amphetamine in either the home (dark squares) or the novel (dark circles) condition. The triangle symbols show the response to control injections of saline. The graph in (b) shows the psychomotor response by rats that received 14 injections of amphetamine (results for first and last injection only are shown) in either the home (dark squares) or the novel (dark circles) condition, or after a 6- to 8-hr habituation to the novel environment prior to each injection (open circles). The triangle symbols show the response to control injections of saline. The graph in (a) is modified from Crombag, Badiani, Maren, and Robinson (2000) and has been adapted with permission of Elsevier. The graph in (b) is modified from Crombag et al. (2001) and has been adapted with permission of Nature Publishing Group.

and relatively novel test cage (novel condition). (Note that the home and novel cages are physically identical, and the only difference between these conditions is in the relative novelty of the test environment where the rats receive drug treatments.) This simple environmental manipulation has a large effect on the ability of drugs to induce psychomotor sensitization, that is, the progressive increase in psychomotor activity produced by repeated drug treatment. Amphetamine, cocaine, and morphine produce much more robust sensitization when administered in the novel condition than when administered at home (Badiani, Browman, \& Robinson, 1995; Badiani, Oates, \& Robinson, 2000). Indeed, when all injection-related stimuli are eliminated by injecting the drug intravenously using remotely controlled pumps (i.e., injections are unsignaled), low to moderate doses of amphetamine given at home often fail to produce sensitization altogether (Browman, Badiani, \& Robinson, 1998; Crombag et al., 2001; Crombag, Badiani, Maren, \& Robinson, 2000; Crombag, Badiani, \& Robinson, 1996). The results from one of these studies are shown in Figure la (note the progressive increase in psychomotor activation produced by amphetamine injections in the novel condition, but not in the home condition).

In follow-up studies, we found that environmental context does not determine sensitization in an all-or-none fashion but shifts the dose required to produce sensitization. That is, when the dose is increased sufficiently, amphetamine and cocaine induce sensitization even at home (Browman et al., 1998).

\section{Is It Conditioning?}

How does this simple environmental manipulation exert such a profound effect on the susceptibility to psychomotor sensitization? One possibility is that when a drug is administered in a distinct test environment (the novel condition), that environment predicts the drug injection (i.e., becomes a conditioned stimulus) and reliably signals the experience of the drug's effects. In contrast, the home environment does not reliably signal drug administration because the rat lives there $24 \mathrm{hr}$ a day. In other words, the differences in sensitization in the home and novel conditions might be due to facilitation of associative learning in the novel environment (Badiani, Browman, \& Robinson, 1995; Crombag et al., 2000). ${ }^{2}$ However, experiments do not support this interpretation. For example, drug treatment can be made

${ }^{2}$ For instance, some researchers have argued that through repeated pairing of the novel environment with the drug effects, environmental stimuli could acquire conditioned stimulus properties and thus the ability to elicit a conditioned "druglike" response. In this view, sensitization occurs because with repeated drug treatments the progressively increasing conditioned response adds to the unchanging unconditioned psychomotor drug effects. 
predictable in the home condition by presenting discrete cues that predict administration of the drug (e.g., lights, tones), and this results in drug-environment conditioning, but has no influence on the development of sensitization (Crombag et al., 2000). Furthermore, degrading the predictive value of a novel environment by habituating the animals to it (i.e., keeping them there at times when the drug is not administered) eliminates drug-environment conditioning, but has no effect on the ability of the "novel" environment to promote sensitization (see Fig. $1 \mathrm{~b}$-note that the response curve of the habituation condition is parallel to that of the novel condition, indicating an identical rate of sensitization).

\section{Is It Stress?}

Another possibility is that the novel environment facilitates sensitization because it acts as a stressor. Novel environments produce stresslike physiological and behavioral responses, and stressors enhance the behavioral and neurobiological responses to drugs. But when this hypothesis was tested, either by manipulating stress mechanisms surgically (by removing the adrenal glands, thereby preventing the release of "stress hormones" such as corticosterone; Badiani, Morano, Akil, \& Robinson, 1995) or by habituating rats to the novel environment and making it less novel (see Fig. 1b; Crombag et al., 2001), sensitization was not affected. Habituation did, however, change the response to the first injection of the drug in the novel environment (Fig. 1b), as did adrenalectomy (Badiani, Morano, et al., 1995), suggesting that the effect of environment on the initial drug response is dissociable from its effects on sensitization.

\section{ENVIRONMENT MODULATES DRUG EFFECTS IN THE BRAIN}

Thus, relatively subtle variations in the environmental context in which drugs are repeatedly administered profoundly alter their longterm behavioral effects, and this is not readily accounted for by some immediate effect of the environment on stress or associative learning processes. Of course, stress and associative learning do have large effects on the actions of drugs, but it seems these processes do not account for the home-novel difference in sensitization.

Whatever the exact "psychological" difference between these two environments, environmental context has a profound effect on the neurobiological impact of drugs like amphetamine and cocaine, and this neurobiological effect may be directly responsible for the effect of environmental context on sensitization. How context influences the neurobiological actions of drugs has been studied by looking at activation of a class of genes in the brain, immediate early genes. The best known immediate early gene, c-fos, is readily activated by many forms of stimulation, including drugs, and because its activation is strongly correlated with neuronal activity, it provides a simple and sensitive indicator of where in the brain neurons are active.

Figure 2 shows the pattern of c-fos activation from one study in which rats were given a single injection of amphetamine or a control saline solution in a home or novel environment. It is obvious that the patterns of brain activation produced by amphetamine (i.e., areas where c-fos is activated following an injection of amphetamine) are very different depending on where the drug is administered. Three findings are particularly interesting: First, a novel environment by itself was sufficient to increase c-fos activation, but this activation occurred primarily in the outer layers of the brain (i.e., in cerebral

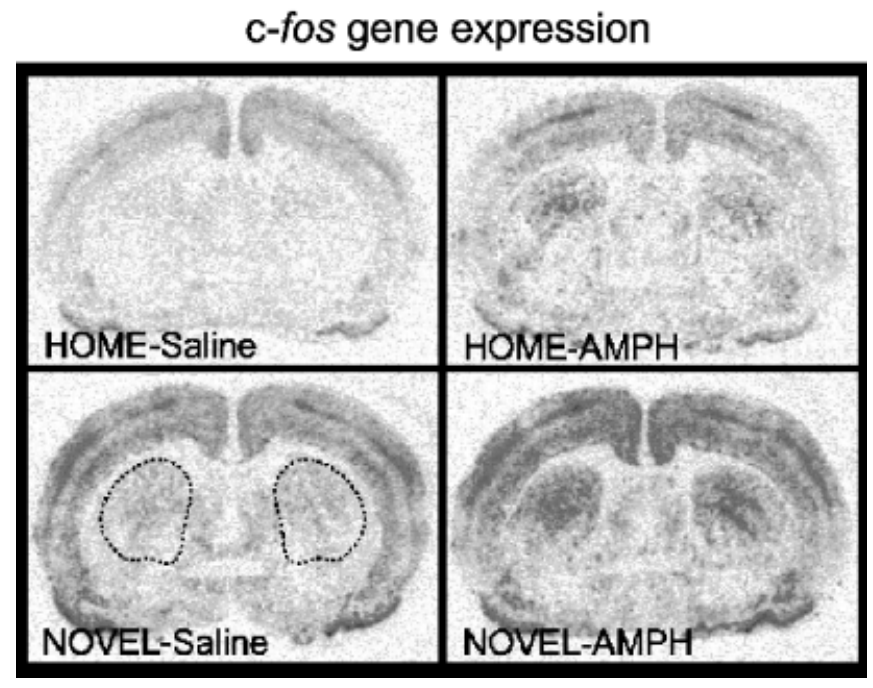

Fig. 2. Levels of c-fos activation in the brain after a single injection of amphetamine or saline solution in the home and the novel condition. The dashed outlines show the region of interest, the striatum, in both hemispheres. Darker shading indicates higher levels of c-fos activation. Photomicrograph provided by Susan M. Ferguson.

cortex; compare the saline response in the home vs. novel conditions). Second, the drug-environment interaction is most apparent in the areas of the brain that are critical for the psychomotor and rewarding effects of drugs, such as the dorsal striatum (compare the amphetamine response in the home vs. novel conditions). Third, in follow-up studies we have found that these differences are also present at the cellular level, in that the specific types of neurons that are activated by amphetamine or cocaine differ depending on environmental context (Badiani et al., 1998; Uslaner et al., 2001).

Although much work remains to be done, it is clear that the neuronal circuitry activated by drugs is powerfully modulated by changes in the environmental context in which the drugs are experienced. Presumably, these neurobiological effects contribute to the ability of drugs to sensitize brain regions that are involved in incentive motivational processes, and therefore play a role in the transition from casual drug use to the compulsive drug-seeking and drug-taking behavior seen in addiction (Robinson \& Berridge, 2003).

\section{ENVIRONMENTAL TRIGGERS OF RELAPSE}

From a clinical perspective, understanding the factors involved in relapse in addicts is critically important. One reason for high rates of relapse may be that sensitization-related changes in brain reward systems are very persistent, if not permanent, rendering addicts highly susceptible to relapse even after long periods of abstinence (Robinson $\&$ Berridge, 2003). In addition, through associative learning processes, environmental stimuli (e.g., drug paraphernalia, drug-related environments) acquire the ability to activate these sensitized reward circuits, and become powerful incentives themselves.

In most animal studies, relapse is modeled using reinstatement procedures. In the first phase of these studies, an intravenous injection of a drug rewards rats for pressing a lever, and like many other species, rats learn to self-administer addictive drugs avidly. 


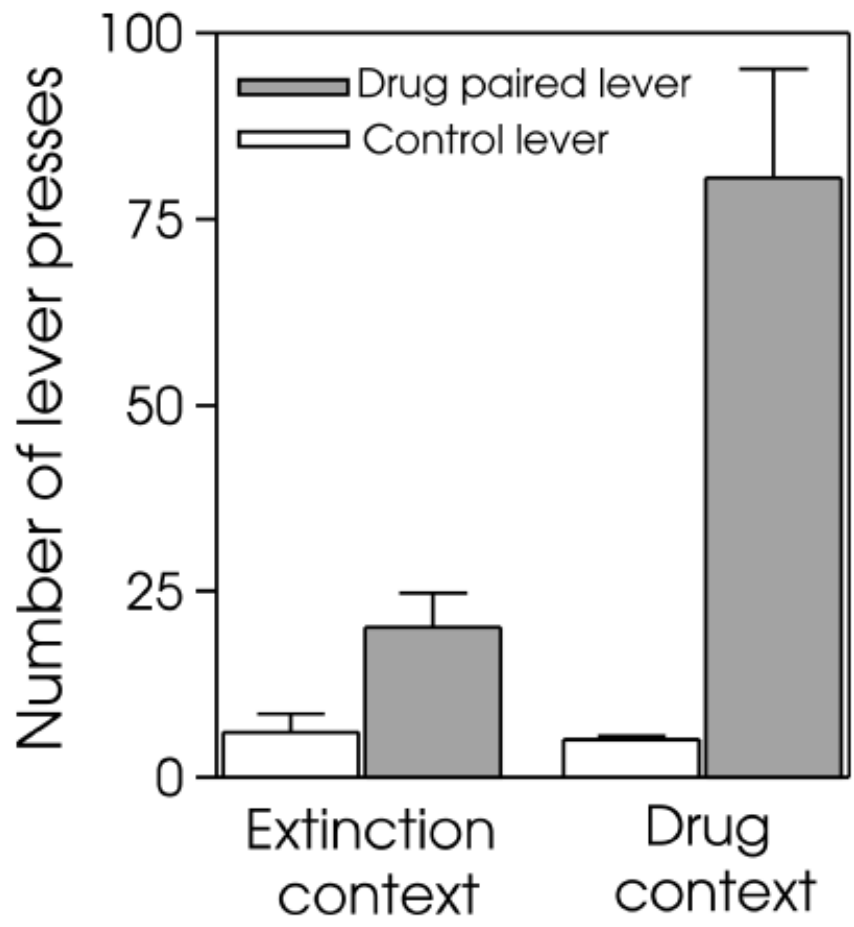

Fig. 3. Results of a reinstatement study with rats. The graph shows the number of lever presses made by the rats in a 2 -hr session when they were either left in the extinction context or placed in the drug-associated context. Responding on a control lever that was not associated with delivery of the drug is shown for comparison. Modified from Crombag and Shaham (2002).

Subsequently, the rats undergo extinction training sessions in which lever presses no longer result in the drug injection. Under these conditions, they typically show an initial burst in responding (called an extinction burst), followed by a steady decline in responding over test sessions. After extinction, lever-pressing behavior can be reinstated by drugs, by stressors, and also by environmental stimuli that have been associated with drug taking. In these types of experiments, the rate (and pattern) of responding observed under (nonreward) extinction conditions is typically used as a measure of drug seeking in rats.

For instance, in two experiments (Crombag, Grimm, \& Shaham, 2002; Crombag \& Shaham, 2002), when rats that had undergone extinction were reexposed to environmental stimuli associated with cocaine or speedball (a cocaine-heroin mixture), their rate of lever pressing returned to what it had been before extinction training (Fig. 3). Furthermore, this effect of environmental context was not seen when rats were pretreated with compounds that block the action of dopamine (Crombag et al., 2002). The fact that environmental stimuli associated with drug taking can activate brain reward circuits and trigger drug seeking may explain why relapse in humans is the rule, rather than the exception (and why contextual factors are so critical in relapse).

\section{CONCLUSIONS AND ISSUES}

In this brief review, we have emphasized that the context in which drugs of abuse are experienced has a large effect on their behavioral and neurobiological effects, and that drug-environment interactions powerfully modulate sensitization, which is thought to be involved in the transition to addiction. In addition, because environmental stimuli associated with drug taking can activate sensitized reward systems directly, they may contribute in a critical way to relapse.

Of course, many questions remain. For example, in all our studies on sensitization, we studied only the psychomotor-activating effects of drugs, and it is not known to what extent other drug effects are influenced in a similar manner. Paolone, Burdino, and Badiani (2003) reported recently that the home-novel manipulation modulates the development of sensitization to the psychomotor-activating effects of morphine, but does not modulate morphine's appetite-facilitating (prophasic) effects or analgesic effects. In addition, the lethal effects of high doses of cocaine are reduced when the drug is administered under novel conditions, relative to the home condition (Browman et al., 1998). Thus, not all drug effects and not all forms of drugexperience-dependent plasticity are modulated by environmental context in the same way. It will be especially important to directly determine if environmental context modulates sensitization to the incentive motivational effects of drugs of abuse.

A second issue concerns the mechanism (or mechanisms) by which the environment alters the susceptibility to sensitization, both at a behavioral-psychological level and at a neurobiological level. We have shown that drugs produce very different effects on the brain and engage different neural circuits depending on the context in which they are given (Badiani et al., 1998; Uslaner et al., 2001). But how alterations in cells and circuits lead to variation in the lasting effects of drugs on brain function and behavior is unknown. Certainly, the past few decades of research have provided an enormous increase in understanding of how addictive drugs alter neurochemical, cellular, and molecular processes in the brain. However, it now appears that to interpret such experimental findings, it will be important to consider some of the seemingly trivial aspects of the testing procedures that researchers use in their studies. Although behavioral scientists typically assess behavior in distinct and relatively novel test environments, many neuroscientists, for the sake of convenience, treat animals in their home cages. Therefore, it remains to be seen how well research findings on the neurobiology of addictive drugs integrate with those on the behavioral effects of these drugs.

The evidence reviewed here suggests that to fully understand the immediate and long-term consequences of addictive drugs on behavior and the brain will require a better appreciation of the environmental circumstances in which drugs are experienced. Indeed, when considering the problem of addiction, it is important to realize that although a large number of people experiment with potentially addictive drugs at some time, few develop an addiction. In other words, addiction is not an inevitable consequence of the mere self-administration of a potentially addictive drug. We suggest that complex interactions between drugs and environmental factors determine the likelihood that addictive drugs produce persistent alterations in neural circuits that contribute to the susceptibility to addiction and relapse.

\section{Recommended Reading}

Anagnostaras, S.G., \& Robinson, T.E. (1996). Sensitization to the psychomotor stimulant effects of amphetamine: Modulation by associative learning. Behavioral Neuroscience, 110, 1397-1414. 
Falk, J.L., \& Feingold, D.A. (1987). Environmental and cultural factors in the behavioral action of drugs. In H.Y. Meltzer (Ed.), Psychopharmacology: The third generation of progress (pp. 1503-1510). New York: Raven Press.

Shalev, U., Grimm, J.W., \& Shaham, Y. (2002). Neurobiology of relapse to heroin and cocaine seeking: A review. Pharmacological Reviews, 54, $1-42$.

Stewart, J., \& Badiani, A. (1993). Tolerance and sensitization to the behavioral effects of drugs. Behavioural Pharmacology, 4, 289-312.

Acknowledgments-We thank Susan M. Ferguson for providing the photomicrographs in Figure 2 and the many colleagues, in particular, Aldo Badiani and Yavin Shaham, who participated in the work summarized in this review.

\section{REFERENCES}

Badiani, A., Browman, K.E., \& Robinson, T.E. (1995). Influence of novel versus home environments on sensitization to the psychomotor stimulant effects of cocaine and amphetamine. Brain Research, 674(2), 291-298.

Badiani, A., Morano, M.I., Akil, H., \& Robinson, T.E. (1995). Circulating adrenal hormones are not necessary for the development of sensitization to the psychomotor activating effects of amphetamine. Brain Research, 673(1), 13-24.

Badiani, A., Oates, M.M., Day, H.E., Watson, S.J., Akil, H., \& Robinson, T.E. (1998). Amphetamine-induced behavior, dopamine release, and c-fos mRNA expression: Modulation by environmental novelty. The Journal of Neuroscience, 18, 10579-10593.

Badiani, A., Oates, M.M., \& Robinson, T.E. (2000). Modulation of morphine sensitization in the rat by contextual stimuli. Psychopharmacology, 151(2-3), 273-282.
Browman, K.E., Badiani, A., \& Robinson, T.E. (1998). The influence of environment on the induction of sensitization to the psychomotor activating effects of intravenous cocaine in rats is dose-dependent. Psychopharmacology, 137(1), 90-98.

Crombag, H.S., Badiani, A., Chan, J., Dell'Orco, J., Dineen, S.P., \& Robinson, T.E. (2001). The ability of environmental context to facilitate psychomotor sensitization to amphetamine can be dissociated from its effect on acute drug responsiveness and on conditioned responding. Neuropsychopharmacology, 24, 680-690.

Crombag, H.S., Badiani, A., Maren, S., \& Robinson, T.E. (2000). The role of contextual versus discrete drug-associated cues in promoting the induction of psychomotor sensitization to intravenous amphetamine. Behavioural Brain Research, 116, 1-22.

Crombag, H.S., Badiani, A., \& Robinson, T.E. (1996). Signalled versus unsignalled intravenous amphetamine: Large differences in the acute psychomotor response and sensitization. Brain Research, 722(1-2), 227-231.

Crombag, H.S., Grimm, J.W., \& Shaham, Y. (2002). Effect of dopamine receptor antagonists on renewal of cocaine seeking by reexposure to drug-associated contextual cues. Neuropsychopharmacology, 27, 1006-1015.

Crombag, H.S., \& Shaham, Y. (2002). Renewal of drug seeking by contextual cues after prolonged extinction in rats. Behavioral Neuroscience, 116(1), 169-173.

Kelleher, R.T., \& Morse, W.H. (1968). Determinants of the specificity of behavioral effects of drugs. Ergebnisse der Physiologie, 60, 1-56.

Paolone, G., Burdino, R., \& Badiani, A. (2003). Dissociation in the modulatory effects of environmental novelty on the locomotor, analgesic, and eating response to acute and repeated morphine in the rat. Psychopharmacology, $166(2), 146-155$.

Robinson, T.E., \& Berridge, K.B. (2003). Addiction. Annual Review of Psychology, 54, 25-53.

Uslaner, J., Badiani, A., Day, H.E., Watson, S.J., Akil, H., \& Robinson, T.E. (2001). Environmental context modulates the ability of cocaine and amphetamine to induce $\mathrm{c}-\mathrm{fos} \mathrm{mRNA}$ expression in the neocortex, caudate nucleus, and nucleus accumbens. Brain Research, 920(1-2), 106-116. 